\title{
Discussion on Financial Analysis Service Based on Big Data
}

\author{
Yixin Zhang \\ School of International Education, WuHan University of Technology, WuHan, China \\ 750614501@qq.com
}

\begin{abstract}
To explore the financial analysis service in the environment of big data, a financial big data analysis service model is established. First of all, the characteristics of financial big data are described and the financial big data analysis service model is introduced. Then, the application of financial big data analysis service model is analysed. The system development environment is discussed, and the system functions are illustrated, including user management, service management, data analysis and visual analysis four parts. Finally, the system use case is designed, and the use case diagram is discussed. Users can conduct loading of market data, analysis of policy calculation results and so on after logging on the system. The research results show that the financial big data analysis service model can work efficiently in the complex big data environment and optimize the business handling process. It can be seen that the financial big data analysis service model can provide timely and rapid financial decisions to maximize service satisfaction.
\end{abstract}

Keywords: big data; financial big data analysis service model; system function.

\section{Introduction}

Financial big data analysis service is an important direction for the development of financial field in the future [1]. According to a survey released by the Chinese Computer Society, the financial sector ranks second in the use of big data. Currently, commercial banks, securities companies, insurance companies, and other daily operations in the financial sector contribute to the huge number of realtime transactions, and each financial institution needs to be supplemented by a large number of historical data to support decision-making [2]. At the same time, the potential value of big data is obvious for the financial sector. Big data changes the current information structure, allowing financial institutions to collect and analyze customer data [3]. Therefore, in view of the high value and fast passing of financial big data, it is necessary to provide more accurate and timely analysis services.

Compared with traditional financial services, services in big data environment are faced with new problems such as strong data set, dimensionality enhancement and data mining difficulty enhancement [4]. In view of the current situation of large number of users, kinds and services, it is necessary to solve the problem of how to timely analyze the diversified consumer needs of users to provide accurate services to users [5]. Therefore, the financial big data analysis service not only pays attention to the user's personal experience to provide non-standardized accurate services, but also encapsulates the analysis results of big data in the form of services to provide users with a variety of assistant decision-making [6]. As a result, the study of financial big data analysis service has great significance in enhancing service value, improving user experience, mining data value and reducing response time.

First of all, the characteristics of financial big data are described and the financial big data analysis service model is introduced. Then, the application of financial big data analysis service model is analysed. The system development environment is discussed, and the system functions are illustrated, including user management, service management, data analysis and visual analysis four parts. Finally, the system use case is designed, and the use case diagram is discussed. 


\section{Introduction to Financial Big Data}

\subsection{Characteristics of Financial Big Data}

Large amount of data: when the Internet technology has not yet been effectively developed, the traditional information technology-supported financial industry has begun to build information technology, such as: credit management, production systems, performance management, and risk control. These data use the original and ancient methods for offline backup and online operation, and store through the national uniformed database. But at this time, the financial data structure and storage mode is relatively single, after the rapid development of the Internet, the financial industry has appeared electronic banks, channels and other high-quality innovations, and the industry has achieved rapid growth [7]. The precipitated data every year shows exponential growth, which leads to the huge number of financial databases in China.

High data quality: the stability and operation of the financial industry is directly related to the stability of China's economy, and has a great impact on related industries to promote the flow of data to create value. The state has strict regulations on all business data of the financial industry, which makes the data quality of the financial industry the highest required in all industries [8]. After information, it is relying on human power to manually input data into the information system over the years, which will inevitably lead to errors and loss of information due to workload, practice, sense of responsibility of the input staff, and technical level.

\subsection{Financial Big Data Analysis Service Model}

Big data analysis service is a functional entity that discovers potential value in big data analysis. It provides data analysis results to display to users on the basis of processing and analyzing big data, so as to assist decision-making. The whole process inputs big data, and then exports the service with data analysis results as encapsulated content. In addition, the process of big data analysis usually adopts the method of storing first and then analyzing, uses big data processing technology and storage tools to collect and preprocess widely heterogeneous data sources, and stores them conveniently according to certain rules. At the same time, for the high value of big data, appropriate processing and mining algorithms and professional calculation methods in related fields are also necessary to complete the analysis work, and finally, through the visualization, the end users can obtain the analysis results intuitively and clearly.

Big data analysis service means that big data service providers encapsulate the analysis results obtained by using big data analysis capability and big data value mining technology to provide services to users. It processes data, analyzes and calculates data according to relevant technical algorithms, and provides the service of data analysis results when users call. There are two service modes: online big data analysis service mode and offline big data analysis service mode, as shown in Figure 1 (a) and (b).

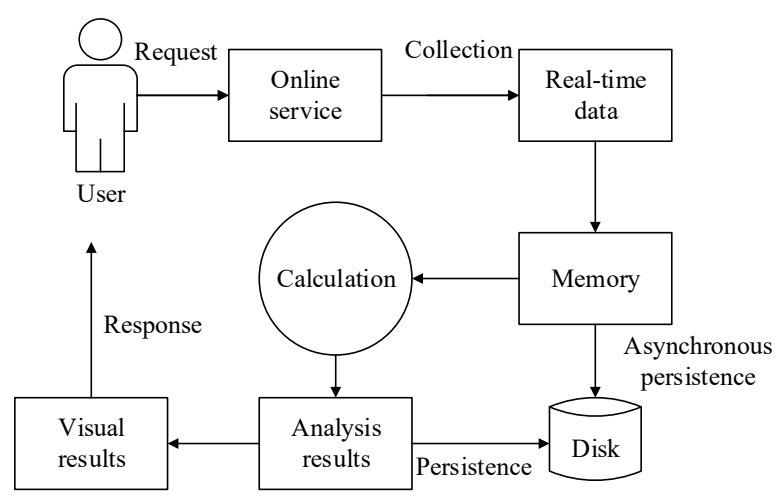

(a) Online big data service mode 


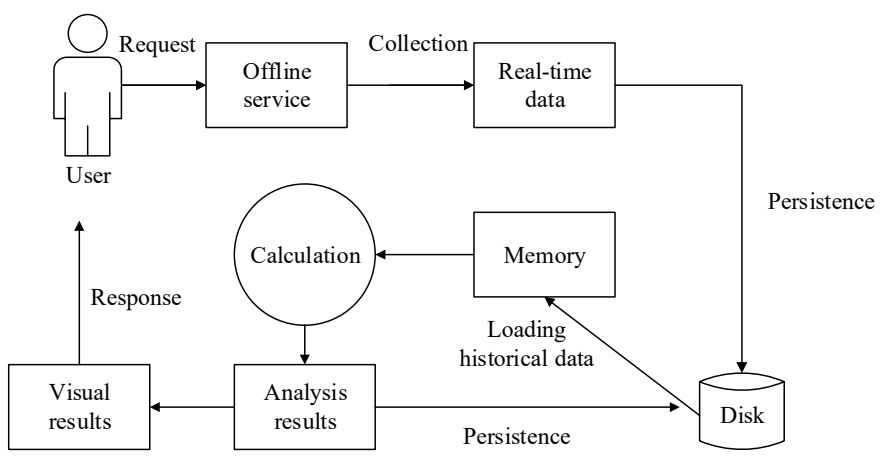

(b) Offline big data service mode

Figure. 1 Two modes of big data service

As can be seen from Figure 1 (a), online big data service requests real-time services, referring to the processing of real-time data collected continuously, which is suitable for high real-time requirements and data throughput slightly smaller scene. In this mode, data entry into the system is generally not stored, but directly calculated in memory results, and finally returned to the user interface in a visual form. At the same time, as can be seen from Figure 1 (b), the offline big data service request mode is to store the real-time data and historical data first, and then re-read the data to calculate when needed, which is more suitable for the scene with low real-time requirements and deep data mining.

The mathematical model of big data analysis service can be divided into 4 levels. Among them, the user requirements layer refers to: there are many users, the analysis needs of multiple users are divided into multiple categories, and the mapping relationship with the next layer is that multiple user analysis needs correspond to a service; the service layer consists of two parts: analysis service composition layer and original sub-service layer. The services in the analysis service composition layer can be composed of many different original sub-services, and the services in the original subservice layer can perform an independent function. The mapping relationship between the services in the analysis service composition layer and the next layer is as follows: one analysis service composition corresponds to a directed acyclic graph computing task; the task layer refers to: a computing task contains multiple data analysis algorithms, and the mapping relationship between the algorithm and the next layer set is: a directed acyclic graph computing task can be assigned to multiple device resources to execute; the device layer refers to the device resources with different memory and CPU computing capability.

\section{Results and Discussion}

\subsection{System Development Environment}

Operating System: Under Windows XP and Linux operating system, all function modules and code of the system are written.

Development environment: The design of the system is divided into two aspects. On the one hand, it is to deal with the data processing commands received and perform data analysis operations through specific data structure processing. On the other hand, it is to complete displaying user operation interface and to verify the rationality of interactive data on the interface. The front-end development environment of the system is Qt Creator 3.1.0 and MFC, the backend is the development environment based on Microsoft Visual Studio 2010. Qt is a cross-platform development framework, suitable for $\mathrm{C}++$ image user interface applications, which can provide all the functions that the image user interface requires for application developers. Microsoft Visual Studio 2010 is a tool that can integrate applications server, database and $\mathrm{C}++$ development, which is also a conveniently used back-end processing development environment.

Development framework: The platform follows MVC framework on the whole, referring to model, view, and controller, respectively. It can map the input, processing, and output functions in the same logic graphic user interface. Among them, model is responsible for data representation and business 
rule expression; view is in charge of displaying the processing data flow in applications; controller answers for receiving user requests, calling the model display requests and choosing image to display the analysis results. The system service structure is shown in Figure 2.

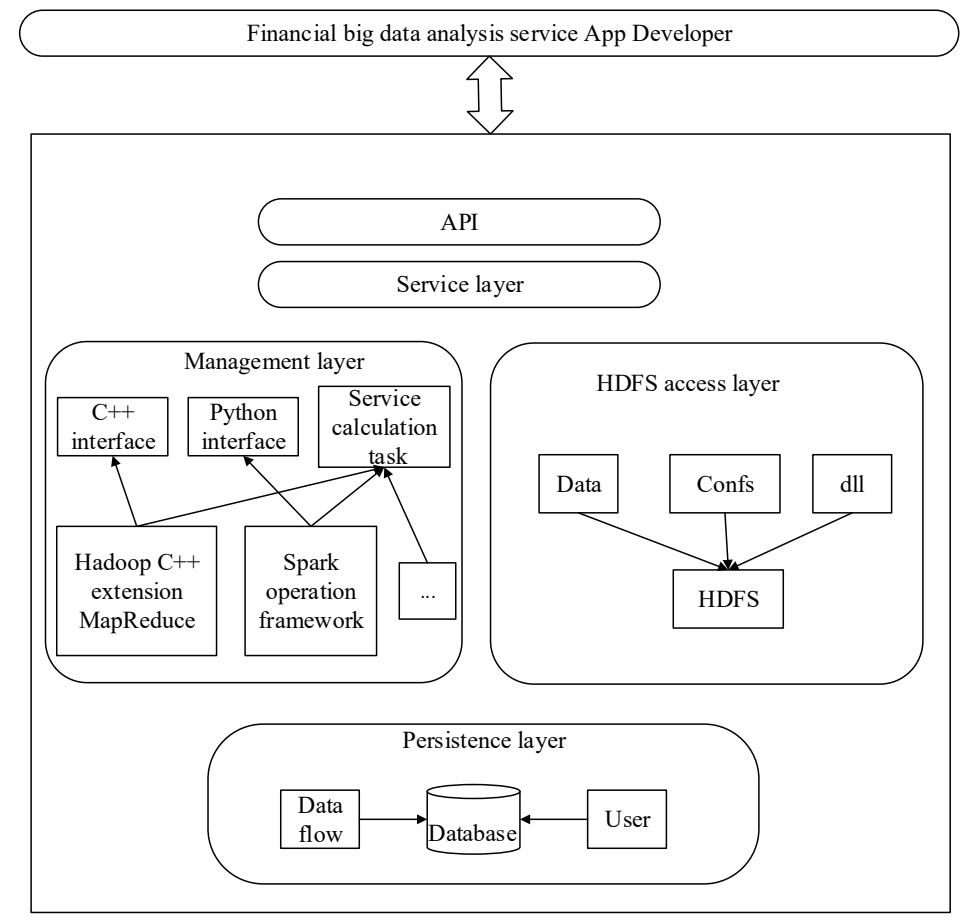

Figure. 2 The system service structure

The application developer of financial big data analysis service accesses the system service by applying API. The service layer mainly translates and executes the data analysis processing flow. The main services of the system are mainly based on three basic service layers: management layer, HDFS access layer and persistence layer. The controller calls the service layer interface and responds to the user request. The system can make users submit multiple tasks in a multi-user and multi-task manner, and multiple tasks can be executed in the background.

\subsection{System Functions}

Through the study of the system model and architecture, the system is divided into four functional modules: user management, service management, data analysis and visual analysis.

Each function module also contains specific sub functions. The main functions of the user management module are: classified users: it analyzes the types of users involved in big data analysis services in the securities field, such as long-term, medium-term and short-term customers. When designing the functions of the system, user classification management should deal with the different needs of ordinary users, experts and programmers, and effectively correspond to different user functions. User information: the basic input of user information is carried out. Role management: the system controls different roles through access control, and classifies customers with the same access rights into one class. Access control: it refers to users with different privileges who login different user interfaces and control with different functions as well as log audit facilitating user processing.

Service management mainly contains service information, composition management and computing strategy management. Service information: the concrete implementation of the service layer in this model. Composition management: the analysis service composition and prediction model can be established. At the same time, it can not only provide composition template through historical data learning, but also add or delete composition artificially. Computing strategy: through the stock strategy method, the valuation of each stock can be calculated. At the same time, by sieve order and ranking increase, stock code and computing strategy, it is possible to quickly assist the user to make decisions and buy. 


\subsection{Use Case Design}

Users mainly use the functions such as display, query, analysis and sorting provided by the system to analyze services. Users only interact with the system through the user interface module, and then they can load the market data and analyze the results of strategy calculation after login, as shown in Figure 3 below.

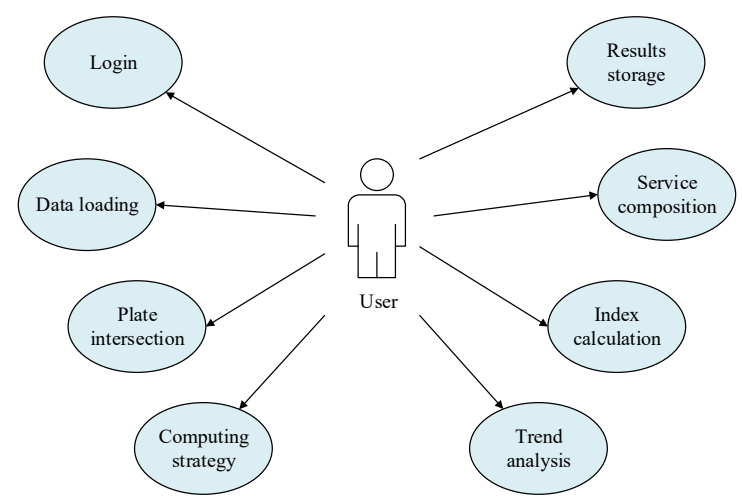

Figure. 3 The use case diagram

The system consists of three main parts: data loading, plate intersection and strategy calculation. Data loading refers to reading the current real-time stock quotation data and displaying them on the interface. Plate intersection is to analyze the demand of different users for different plates, and to classify different stocks according to the plates. Strategy calculation is the stock and strategy selection for multiple users. Through calculation and analysis of historical data, the strategy value is obtained to provide decision-making services for users.

\section{Conclusion}

In the era of big data, the development of financial data analysis services is facing many new challenges. Research on a service system that supports securities users to make decisions and forecasts faster and better can meet the needs of users, and reflect the value and timeliness of the service, respectively. Starting from the features of financial big data, the financial big data analysis service system model is explored, aiming at building a high-value and efficient service application in the financial big data environment. Then, the system development environment, system functions and user case of financial big data analysis service model are described. The results show that the information extracted and analyzed from the big data can provide effective decision-making service support for users through relevant methods and techniques.

However, financial big data analysis services are still faced with the following two problems. From the perspective of value, in view of the great potential value of financial data and difficulty to obtain, how to fully obtain the value of useful information in the data is a problem that needs to be explored in depth. From the point of view of speed, in terms of the large amount of financial historical data and the large number of analysis and measurement indicators, how to deal with the analysis data promptly and quickly and whether it can meet the time tolerance in the financial field is a problem to be solved.

\section{References}

[1]. Raghupathi W, Raghupathi V. Big data analytics in healthcare: promise and potential. Health information science and systems, 2014, 2(1), pp. 3.

[2]. Chong G U O. Modeling and Simulation of Online Customer Loyalty Based on Analysis of Big Data. Computer Simulation, 2015, 10, pp. 053. 
[3]. Grover V, Chiang R H L, Liang T P, et al. Creating Strategic Business Value from Big Data Analytics: A Research Framework. Journal of Management Information Systems, 2018, 35(2), pp. 388-423.

[4]. Demirkan H, Delen D. Leveraging the capabilities of service-oriented decision support systems: Putting analytics and big data in cloud. Decision Support Systems, 2013, 55(1), pp. 412-421.

[5]. Vickery S K, Jayaram J, Droge C, et al. The effects of an integrative supply chain strategy on customer service and financial performance: an analysis of direct versus indirect relationships. Journal of operations management, 2003, 21(5), pp. 523-539.

[6]. Kwon O, Lee N, Shin B. Data quality management, data usage experience and acquisition intention of big data analytics. International Journal of Information Management, 2014, 34(3), pp. 387-394.

[7]. Frizzo-Barker J, Chow-White P A, Mozafari M, et al. An empirical study of the rise of big data in business scholarship. International Journal of Information Management, 2016, 36(3), pp. 403413.

[8]. Tirunillai S, Tellis G J. Mining marketing meaning from online chatter: Strategic brand analysis of big data using latent dirichlet allocation. Journal of Marketing Research, 2014, 51(4), pp. 463479. 